\title{
Development of an evidence-based decision aid on complementary and alternative medicine (CAM) and pain for parents of children with cancer
}

\author{
Miek C. Jong ${ }^{1} \cdot$ Inge Boers ${ }^{2} \cdot$ Herman van Wietmarschen ${ }^{2} \cdot$ Martine Busch $^{3} \cdot$ Marianne C. Naafs $^{4}$. \\ Gertjan J. L. Kaspers ${ }^{5,6}$ - Wim J. E. Tissing ${ }^{6,7}$
}

Received: 16 April 2019 / Accepted: 28 August 2019 /Published online: 6 September 2019

(C) The Author(s) 2019

\begin{abstract}
Purpose To develop an evidence-based decision aid for parents of children with cancer and to help guide them in the use of complementary and alternative medicine (CAM) for cancer care.

Methods This study had a mixed research design. The needs of parents were investigated by survey and focus group. A systematic review and meta-analysis were performed on the effectiveness of CAM using Grading of Recommendations Assessment, Development and Evaluation (GRADE). Clinical experts were interviewed and a decision aid on CAM treatment for pain was developed.

Results Parents emphasized the importance of reliable information on CAM, focusing primarily on communication and a broad spectrum of complaints related to cancer treatment. The decision aid on CAM for pain included five modalities based on 11 randomized control trials (RCTs): hypnotherapy, mind-body techniques, massage, healing touch, and music therapy. Metaanalysis could be performed on hypnotherapy, which significantly reduced cancer-related procedural pain compared with standard care $(\mathrm{MD},-1.37 ; 95 \% \mathrm{CI},-1.60,-1.15 ; P<0.00001)$ and attention control $(\mathrm{MD},-1.13 ; 95 \% \mathrm{CI},-1.34,-0.94$; $P<0.00001$ ), and massage, demonstrating no effect on pain compared with standard care (MD, $-0.77 ; 95 \%$ CI, $-1.82,0.28$; $P=0.15)$. Research evidence and supplementary information from clinical practice and patient were incorporated in a websitebased decision aid.

Conclusions An evidence-based decision aid was developed to support parents of children with cancer in making decisions about CAM for pain management. Next steps will be to expand the website to include additional childhood cancer-related complaints and to evaluate its use in practice.
\end{abstract}

Keywords Pediatric oncology $\cdot$ Systematic review $\cdot$ Meta-analysis $\cdot$ Hypnotherapy $\cdot$ Procedural pain $\cdot$ Decision-making

Miek C. Jong and Inge Boers contributed equally to this work.

Electronic supplementary material The online version of this article (https://doi.org/10.1007/s00520-019-05058-8) contains supplementary material, which is available to authorized users.

Miek C. Jong

miek.jong@miun.se

1 Department of Health Sciences, Mid Sweden University, Holmgatan 10, 85170 Sundsvall, Sweden

2 Department Nutrition \& Health, Louis Bolk Institute, Kosterijland 3-5, 3981 AJ Bunnik, The Netherlands

3 Van Praag Institute, Springweg 7, 3511 VH Utrecht, The Netherlands
4 Netherlands Childhood Cancer Parent Organization VOKK, Schouwstede 2b, 3431 JB Nieuwegein, The Netherlands

5 Emma Children's Hospital, Amsterdam UMC, Vrije Universiteit Amsterdam, Paediatric Oncology, Cancer Center Amsterdam, De Boelelaan 1117, 1081 HV Amsterdam, The Netherlands

6 Princess Máxima Center for Pediatric Oncology, Heidelberglaan 25, 3584 CS Utrecht, The Netherlands

7 Department Paediatric Oncology, University Medical Center Groningen, Hanzeplein 1, 9713 GZ Groningen, The Netherlands 


\section{Introduction}

Complementary and alternative medicine (CAM) can be defined as healthcare approaches that are not typically part of conventional medical care or that may have origins outside of usual Western practice [1]. Although not part of mainstream medicine, an increasing number of children with cancer use CAM along with conventional therapies [2]. Parents choose CAM for treatment and cure of cancer in their children [3], and also as supportive care agent to help minimize side effects of cancer treatment and to improve the general quality of life and well-being $[4,5]$. The prevalence of CAM use among children with cancer is high and varies between 6 and $100 \%$, depending on the survey sample and country, and is on average $47.2 \%$ in high-income countries [3]. CAM modalities most commonly used are herbs, diets and nutrition, homeopathy, and prayer $[4,6,7]$. Despite the high prevalence of CAM use in children with cancer, it is difficult for parents to find reliable information about it. Existing platforms developed to advise patients on CAM use for cancer, for example the website of the National Cancer Institute [8], are directed towards cancer in adults and do not specifically discuss the suitability of CAM modalities for use in children. Other information available on the internet is of poor quality [9] and frequently includes the statement: "Always consult with your child's physician before beginning any CAM, because some therapies may interfere with standard treatment." However, physicians typically do not receive training in CAM and have limited knowledge on the subject and so find themselves unable to discuss any possible (side) effects of CAM $[10,11]$. Additionally, it is known that few parents even discuss CAM use with the pediatric oncologist $[7,12,13]$. Authoritative and reliable access to information is thus needed to support parents in their search for information on CAM modalities as potential supportive treatment options for their children with cancer.

This study describes the process and outcome of a project that aimed to develop and implement an evidence-based decision aid on CAM use for pain in order to enable parents of children with cancer to make well-informed decisions concerning CAM. Since children with cancer use CAM most often as supportive care agent $[14,15]$, the decision aid focused on supportive CAM care for pain.

\section{Methods}

\section{Project design}

The project had four phases of mixed methodology design (see Fig. 1) and was carried out from 2014 to 2017 by a multidisciplinary team of pediatric oncologists, members of the patient organization for parents of children with cancer (VOKK), CAM experts, and researchers. A scientific advisory board consisting of six pediatricians/pediatric oncologists supported the project team.

\section{Phase 1: needs of parents}

The needs of parents with respect to a decision aid on CAM use for their children with cancer were investigated by means of a survey and focus group.

Survey In April-May 2015, a 23-item anonymous questionnaire was distributed by the VOKK to parents of children with cancer via their e-newsletter and Facebook campaign. The questionnaire was modified from previously used questionnaires on self-management instruments [16] and CAM [7, 17]. It was validated through a pilot survey among eight parents and revised accordingly with respect to rewording terms to prevent misinterpretation. The questionnaire is attached as online resource 1. Descriptive statistics were used to analyze the data by means of SPSS 22.0.

Focus group A focus group took place in June 2015 with seven parents of children with cancer that were recruited by the VOKK. The first part of the focus group used the brainwriting technique [18] in response to the following question: What do you need in order to make a good choice about CAM use for your child? Next, the following topics were discussed: possible reasons to use CAM, information and decision-making on CAM use, communication about CAM with physicians/oncologists, and any additional requirements for a decision aid on CAM. The focus group lasted $2 \mathrm{~h}$ and was recorded, and field notes were taken. A directive content analysis [19] was performed to get a better understanding of the parents' needs regarding a decision aid on CAM.

\section{Phase 2: evidence on CAM}

Evidence for CAM was investigated by means of a systematic review and meta-analyses. It was limited to pain management in children as it was not feasible within the project grant and time to perform additional systematic reviews on other cancerrelated complaints.

Systematic review and meta-analysis The review was planned and conducted in accordance with the Preferred Reporting Items for Systematic Reviews and Meta-Analyses (PRISMA) guidelines [20]. The protocol was not registered in a database. The PICOS (Patient-Intervention-ComparisonOutcome-Study) design for the systematic review was defined as follows: Effects (reduction) on experienced pain during cancer treatment and post-treatment and occurrence of adverse events (outcome) of CAM (intervention) in comparison with no treatment, standard treatment, and placebo control or active control group (comparison) in children with cancer (patient) 

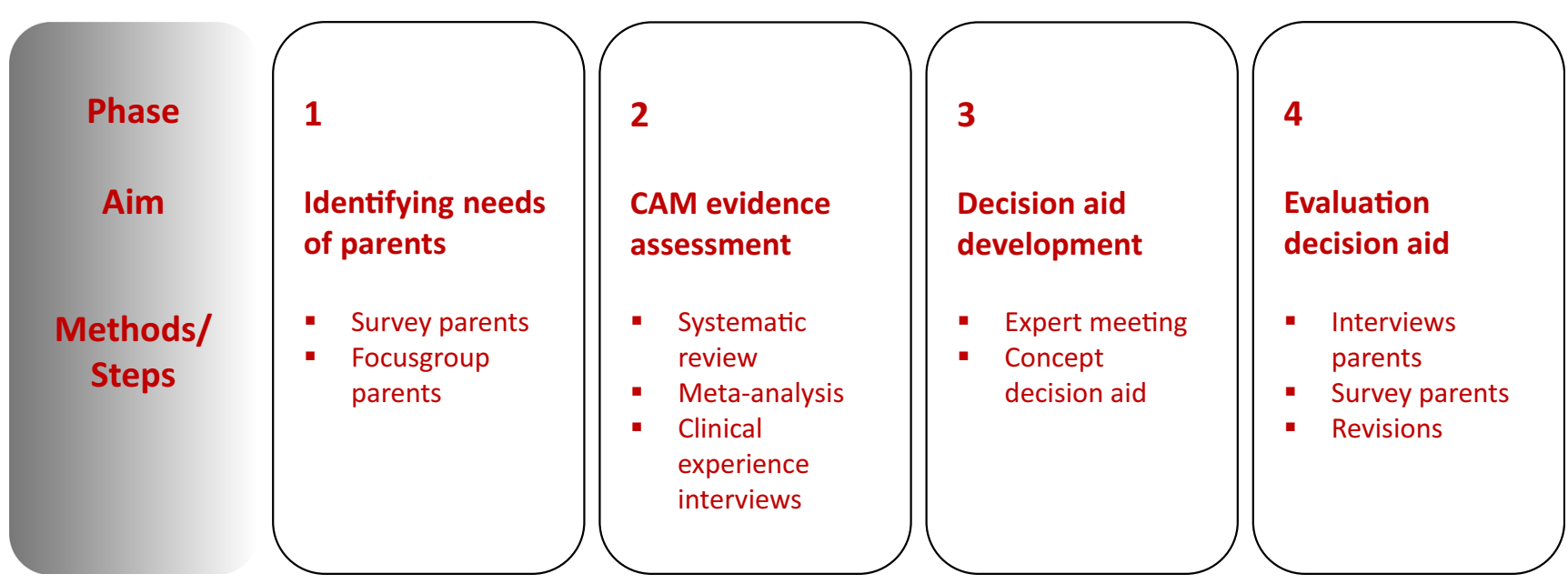

Fig. 1 Project overview

reported in prospective controlled studies (study). Between December 2015 and March 2016, the PubMed, EMBASE, Cochrane, and CINAHL were searched. Search terms and strings are attached as online resource 2. Inclusion criteria were peer-reviewed prospective controlled studies in children (0-18 years) with any type of cancer undergoing a CAM intervention (as defined by the National Center for Complementary and Alternative Medicine [21]) published in English or Dutch language. The primary outcome was pain (any pain-related outcome), and the secondary outcome was reported CAM-related adverse events. Two researchers performed the selection of studies, data extraction, and risk of bias assessments. Any instances of disagreement were resolved by consultation with a third researcher. The risk of bias assessment was done according to the Grading of Recommendations Assessment, Development and Evaluation (GRADE) handbook [22]. The GRADE approach was used to facilitate the overall quality of each outcome and to assess risk of bias across studies [23]. Meta-analysis was performed in cases where the results of two or more randomized clinical trials (RCTs) per CAM modality could be pooled using RevMan 5.3 (Cochrane collaboration) and GRADEpro (version 3.6) for different scores related to pain across interventions. $I^{2}$ was used to test for heterogeneity in judging consistency of evidence.

Clinical experience Six pediatric oncologists working at integrative pediatric oncology clinics in Canada, the USA, Germany, and Italy were interviewed to collect information related to the use and safety of CAM in the treatment of pain in children with cancer. These experts were identified at the International Congress on Integrative Medicine and Health (Las Vegas, 2016). The interview guide consisted of seven questions (see online resource 3). Interviews were conducted face-to-face or via Skype, were recorded, and varied in length from 30 to $60 \mathrm{~min}$. The content was analyzed and categorized as follows: CAM modalities, effects, treatment phase, type of pain and patient, complaints, and side effects.

\section{Phase 3: development decision aid}

The objective of phase 3 was to combine information on research evidence and clinical expertise in order to inform recommendations and decision-making regarding CAM use for pain management in children with cancer. A 3-h expert meeting was organized in June 2016 consisting of a balanced mix of pediatric oncologists, pediatricians, pediatric nurses, parents, CAM experts, researchers, and members of the patient organization. The meeting included 14 participants in total. Preceding the expert meeting, all participants read the outcome and underlying documentation of phase 2 and were informed about the procedure and steps in the meeting, which followed the seven-step approach of GRADE [22]. Other CAM-related contextual factors taken into consideration included whether the CAM treatment was safe, feasible to implement, could be taught and practiced by the children or the parents independently, was practiced individually or as a group, and whether it was a facilitator for conventional treatment. Based on the recommendations and decisions that were reached in the expert meeting and on the results from the earlier phases, three text writers (representing the patient, CAM, and scientific perspective) wrote the content for the decision aid. The concept structure of the decision aid was developed by members of the project team in parallel with technical support from KSMT design (The Hague, the Netherlands).

\section{Phase 4: evaluation of decision aid}

The content and structure of the decision aid were evaluated through interviews and a short questionnaire (see online resource 4) administered to the VOKK, three pediatric 
oncologists, and 13 parents. Evaluation was based on the look and feel, structure, ease of comprehension, usability, navigation, usefulness in decision-making, and interface. Analysis of feedback was performed by one researcher and the content was adapted accordingly.

\section{Results}

\section{Phase 1: needs of parents}

In order to develop a practical and useful decision aid on CAM use that is likely to be adopted by families affected by cancer, the project started with investigation of the needs of the parents.

Survey Among those surveyed, 70 parents responded. Mothers made up the majority of respondents (93\%). Among all respondents, most had a child with leukemia $(55 \%)$ or a brain tumor $(17 \%)$. Among the cancer-affected children of respondents, $31 \%$ were currently in treatment and $38 \%$ had finished treatment more than 1 year ago. More than half $(56 \%)$ reported CAM use for their child. Among those who reported CAM, the use of food supplements/ vitamins (32\%), massage (22\%), and homeopathy (22\%) were most mentioned. One-third of those using CAM for their child had searched for information about the CAM treatment before use. Sources included the Internet (37\%), family/friends (37\%), and CAM practitioners (26\%). Eighty-eight percent of respondents thought it was important to receive/find good quality information on CAM. Most respondents (79\%) had a need for information on CAM following their child's cancer diagnosis, including $38 \%$ just after diagnosis, $45 \%$ during the first year after cancer treatment, $34 \%$ more than a year following cancer treatment, and $14 \%$ in the palliative phase. Parents sought information on CAM for treatment of fatigue (62\%), anxiety (47\%), pain (46\%), weakened immune system (44\%), sleeping problems (42\%), nausea/vomiting (35\%), low mood (29\%), decreased appetite (27\%), intestinal problems (22\%), concentration problems (16\%), and weight loss $(11 \%)$. Parents mentioned a need for a decision aid to support their discussions of CAM treatments with their physician $(60 \%)$, to find reliable CAM practitioners (59\%), for education regarding possible CAM use (45\%), for support in asking questions of CAM experts $(43 \%)$, and as a resource for reading about other parents' experiences with CAM (40\%). Preferences for the form of the decision aid were website (62\%), app (23\%), or informational brochure/leaflet (12\%). Preference of most respondents was that the decision aid be accessible via the treating pediatric oncologist (88\%) or VOKK (83\%).

Focus group Direct content analysis was performed on five themes: (1) Search strategies: Parents usually start searching for CAM options after their child has been receiving treatment for some time as closer to time of diagnosis they have too much information to process. Parents report searching the Internet for CAM modalities effective for specific complaints or cancer types, for finding reliable CAM practitioners, and for information on whether particular CAM modalities can be combined with cancer treatment. (2) Decision-making: Decisions on the use of CAM are mostly based on the positive experiences of family/friends, on parent's personal experiences with CAM, or on the availability of evidence for a particular CAM modality. (3) Format of the decision aid: A website with a search function for complaint, type of cancer, and CAM modality, along with a chat function allowing interaction with other parents and a CAM expert who can answer CAM-related questions. (4) Function of the decision aid: To allow the parents make their own decisions with regard to $\mathrm{CAM}$, and also as a tool to discuss the outcome on CAM with the pediatric oncologist. (5) Content of the decision aid: Description of positive effects of particular CAM modalities, evidence for their use, potential side effects, and possible interference with cancer treatment.

\section{Phase 2: evidence on CAM}

Based on the results of phase 1, the project team needed to make a decision on the focus of the decision aid. Since there are more than 1800 CAM modalities [24] and performing systematic reviews to collect evidence-based information on all these modalities is time-consuming, it was not feasible to develop a decision aid on all childhood cancer-related complaints for which parents in phase 1 wanted information about. It was decided to focus on CAM use for pain during cancer treatment and post-treatment because (1) pain was in the top three complaints that parents wanted information about in phase 1 and (2) the Dutch Childhood Oncology Group (DCOG; www.skion.nl) was developing clinical guidelines for treatment of pain, so a joint venture was planned.

Systematic review on CAM for pain Figure 2 shows the study selection process. A total of 6936 records were identified from searches, of which 11 RCTs met the criteria for GRADE analysis [25-35]. An additional 18 articles served as input for phase 3 (see online resource 5). The characteristics of the included RCTs are summarized in Table 1. RCTs were found for five CAM modalities: hypnotherapy $(N=5)$, massage $(N=3)$, healing touch $(N=1)$, music therapy $(N=1)$, and mind-body intervention $(N=1)$. Ten out of 11 studies investigated the changes in pain related to procedures such as venapuncture, lumbar puncture, or bone marrow aspiration. The 11 studies included children with all types of cancer. The quality of studies was moderate to high for the RCTs on hypnotherapy and low to moderate for the other CAM modalities (Table 1). Since the studies differed with respect to pain scales, control groups, and number of RCTs per 
Fig. 2 Flow diagram of study selection and identification

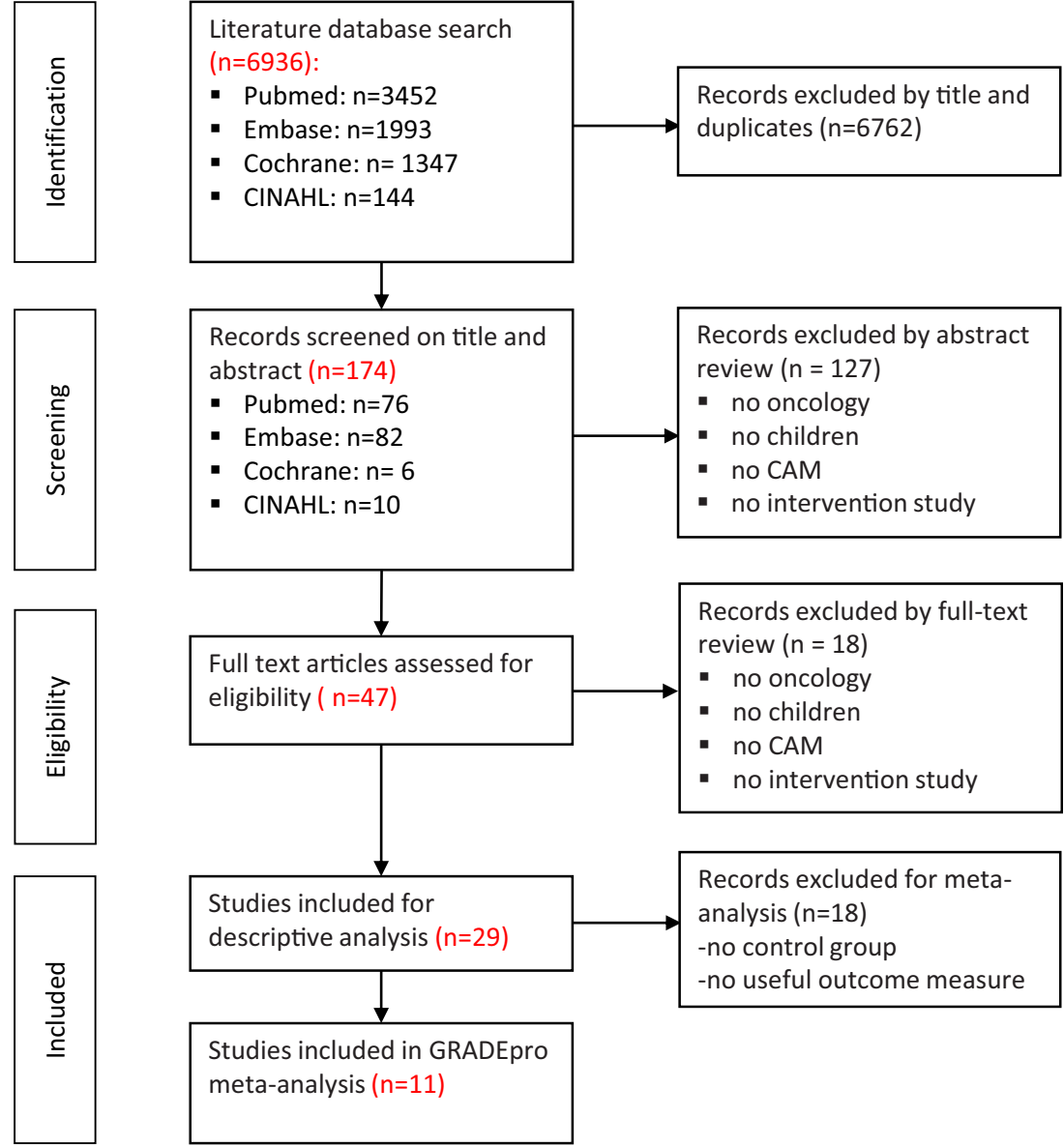

CAM modality, pooled effects on the primary outcome could only be analyzed for hypnotherapy and massage (Table 2). There was high-quality evidence that hypnotherapy significantly reduces cancer-related procedural pain in children; the pooled effect was statistically significant (MD, - 1.37; 95\% CI, - 1.60, 1.15; $P<0.00001)$ compared with standard care [27-29], and statistically significant (MD, $-1.13 ; 95 \% \mathrm{CI},-1.34,-0.94 ; P<$ 0.00001) compared with an attention-control group [26-29] (Table 2). Both analyses showed significant heterogeneities $(P<0.001)$, with $I^{2}$ values of $87 \%$ in comparison with standard care and $86 \%$ in comparison with attention-control. One highquality study on hypnotherapy by Smith et al. [34] was not included in the meta-analysis because it used a different VAS scale and outcome data were only reported for children with high hypnotizability and children with low hypnotizability. There was low-quality evidence for no effect of massage (Swedish/acupressure and effleurage/petrissage techniques) on cancer-related procedural pain compared with standard care; the pooled effect of two studies was not significantly different $(\mathrm{MD},-0.77 ; 95 \% \mathrm{CI}$, $-1.82,0.28 ; P=0.15$, heterogeneity $I^{2}=0 \%, P=0.80$ ) (Table 2). No evidence was found that the five CAM modalities were unsafe to treat pain in children with cancer.

Since it is more challenging to conduct trials in children than in adults [36], especially with regard to cancer, the number of pediatric patients in studies is still limited [37]. Therefore, in order to collect more information on the appropriate use of specific CAM modalities for pain management in children with cancer, it was decided to investigate the clinical experiences of pediatric oncologists with expertise in CAM. Experts had positive clinical experience with all five CAM modalities that were found in the systematic review (see online resource 6).

\section{Phase 3: development decision aid}

Data of the systematic review and the clinical experience from pediatric oncologist, as obtained in phase 2, formed the main input for the development of the decision aid in phase 3. A crucial step in phase 3 was to reach consensus among stakeholders with respect to recommendations on CAM use. Five CAM modalities (hypnotherapy, mind-body techniques, massage, healing touch, and music therapy) were included in the decision aid based on the results from the systematic review. The clinical expert opinions of pediatric oncologists in Integrative Oncology as well as patients' needs and preferences were considered important supplementary sources of information for these five CAM modalities. Recommendations were given for each CAM modality (see Table 3 ). The project team processed the information from the phase 2 literature analysis and expert 


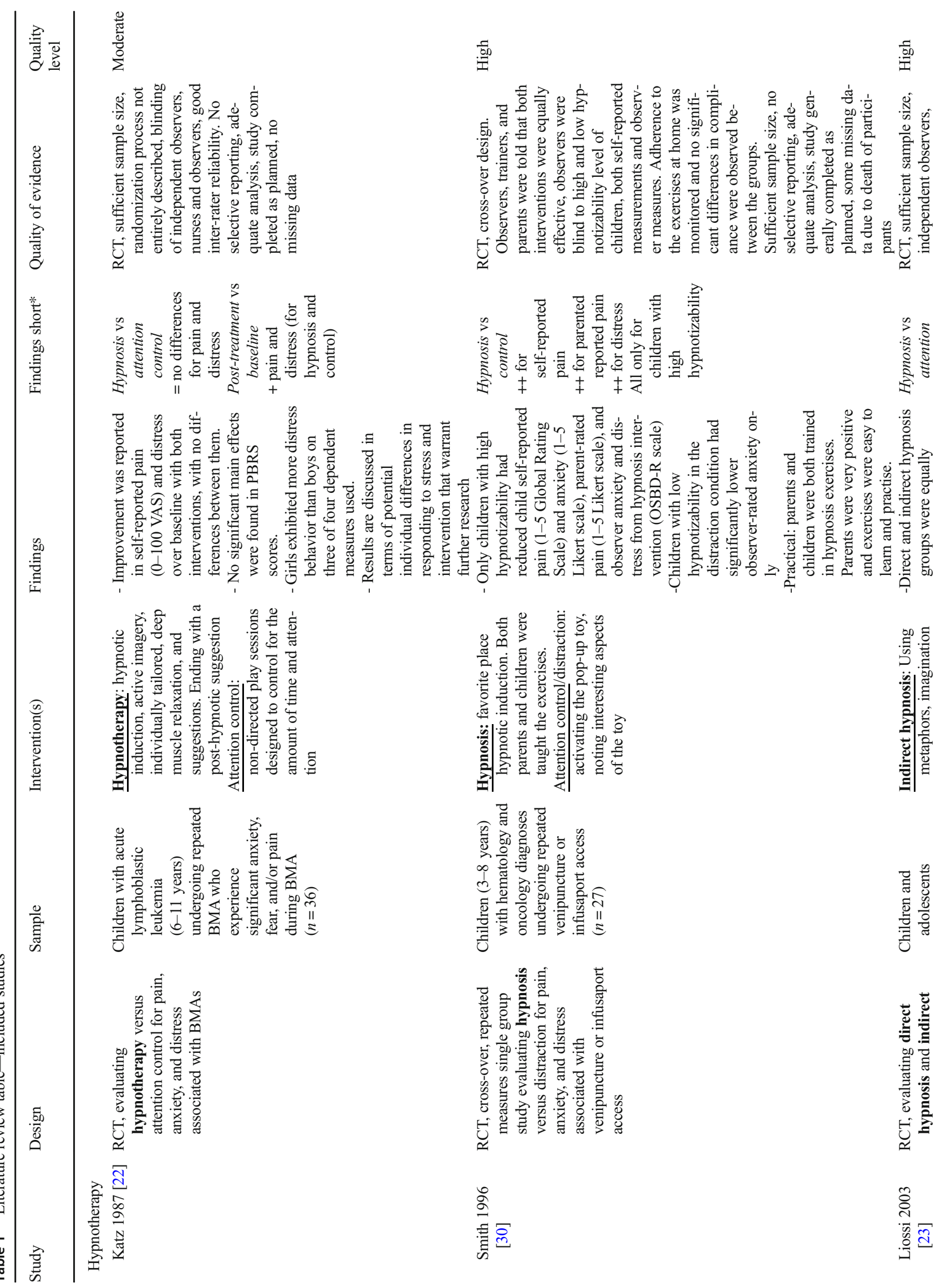




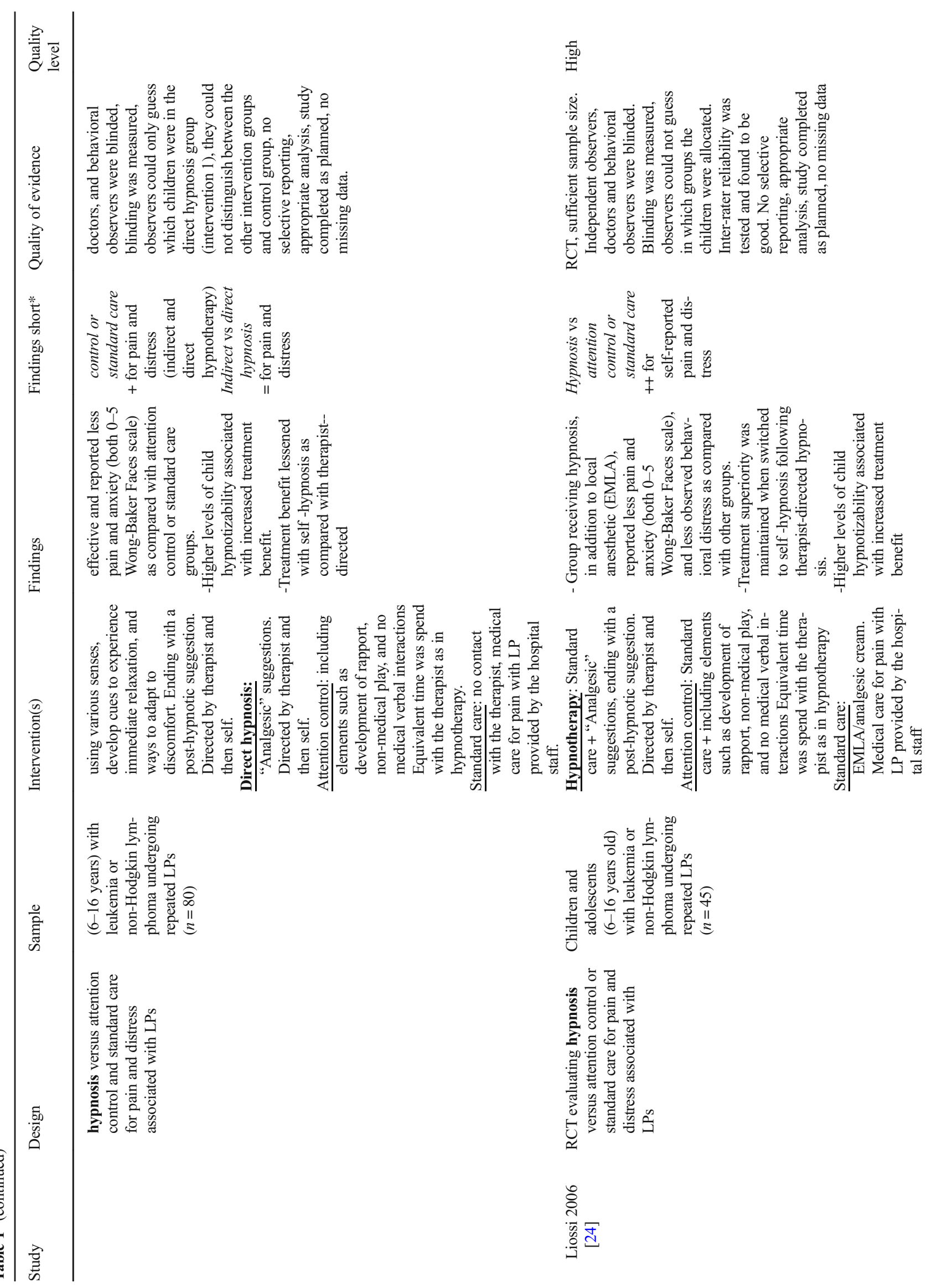




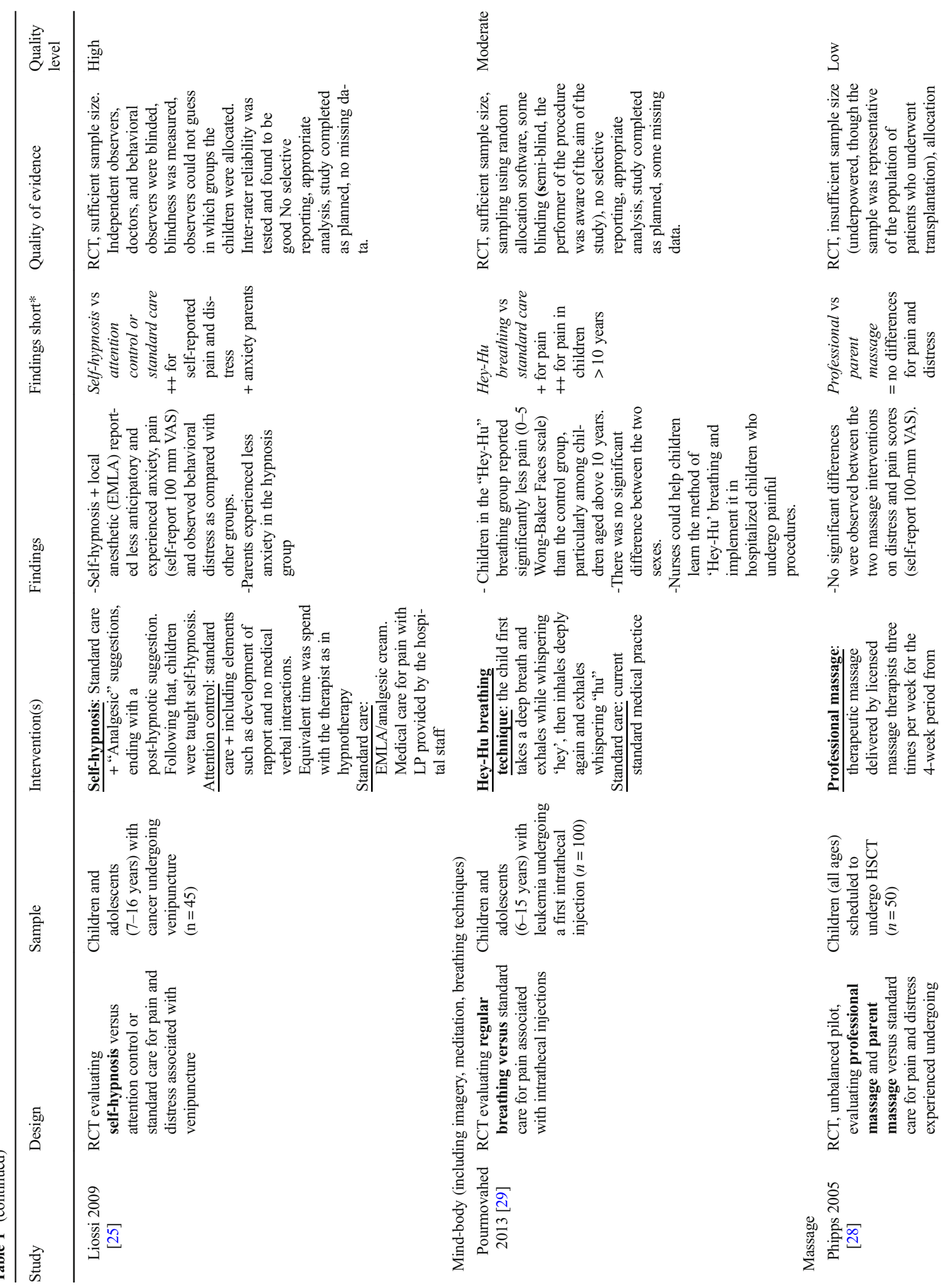




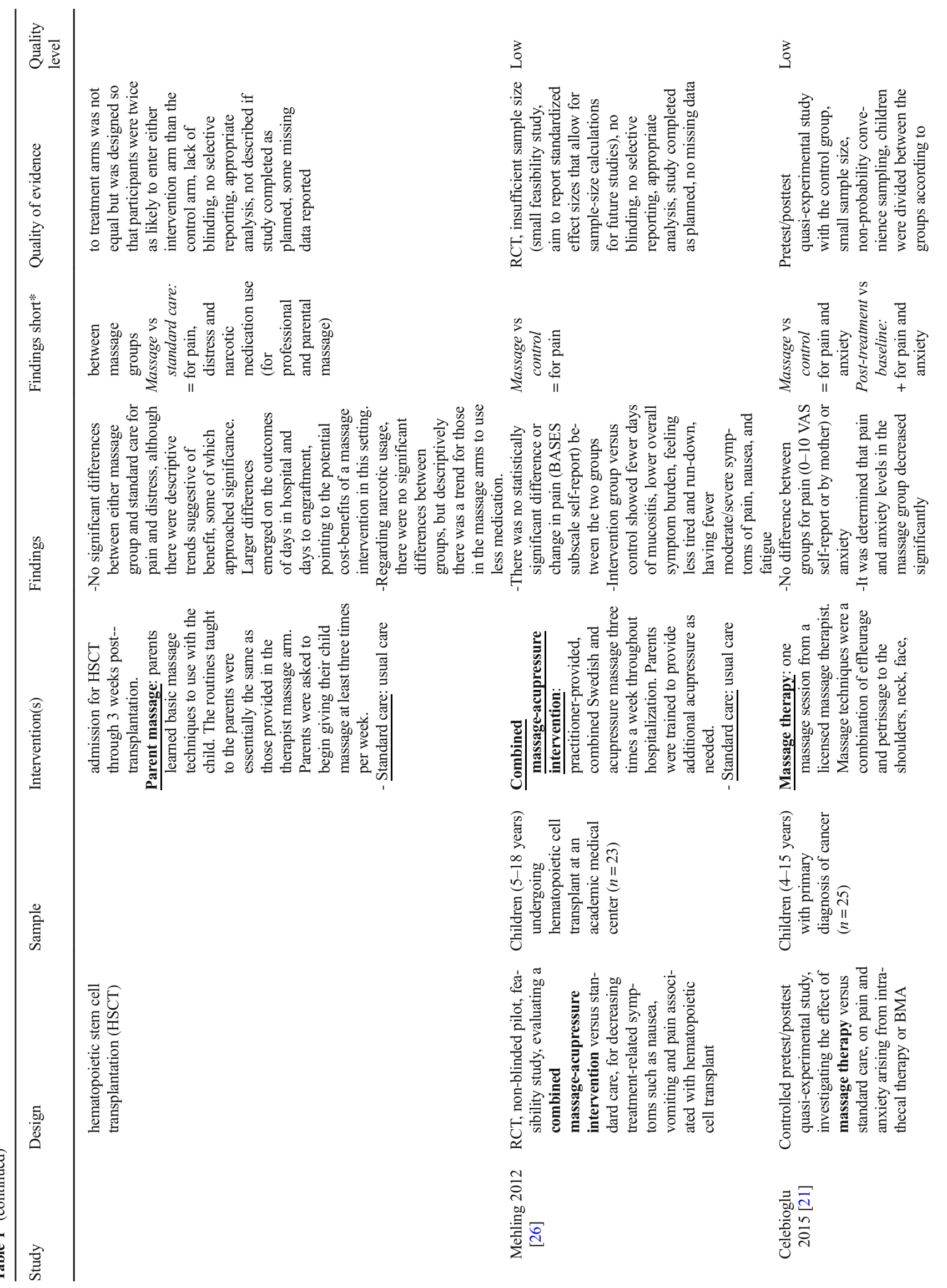




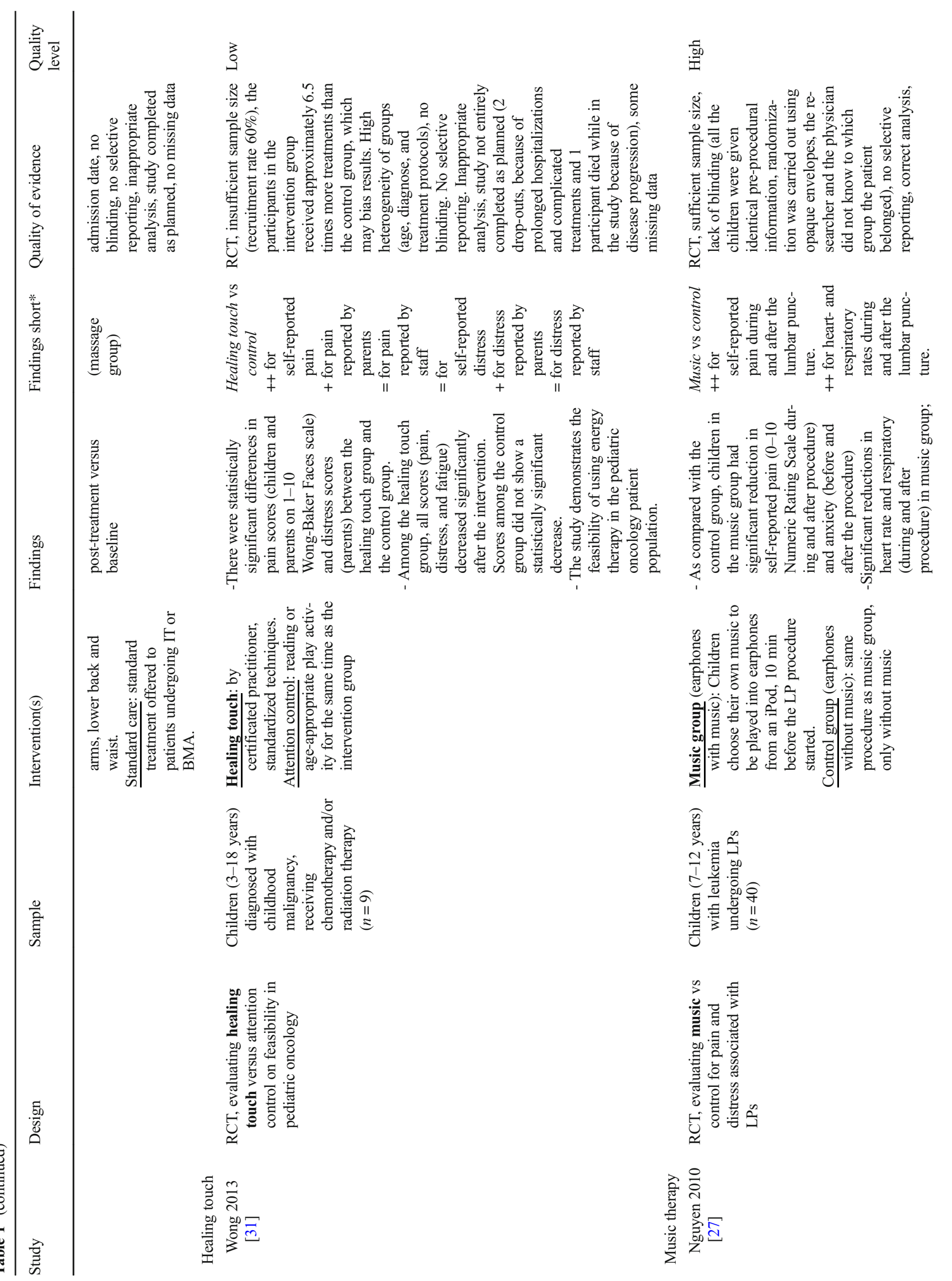




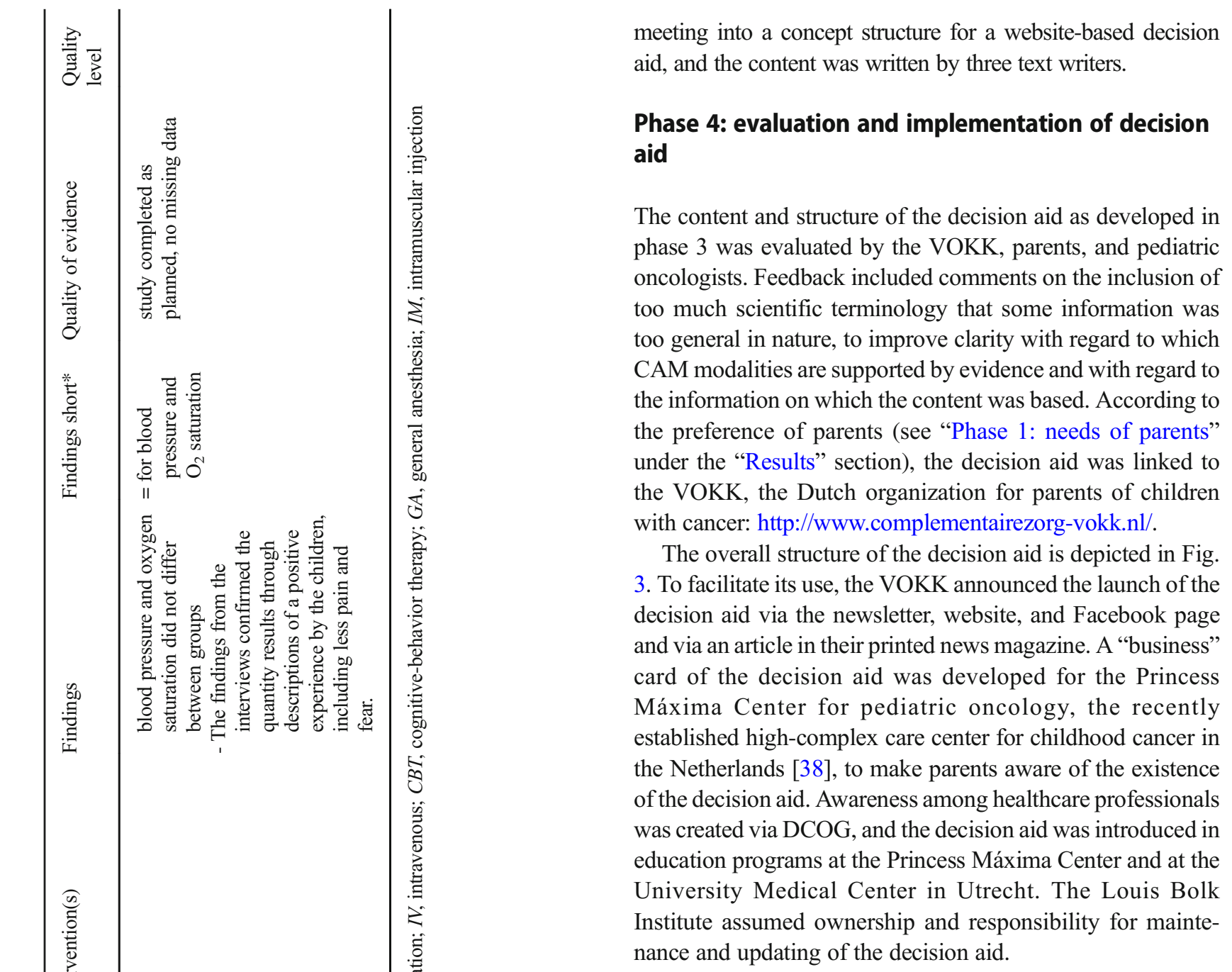

\section{Discussion}

To the best of our knowledge, this is the first study that reports on the development of an evidence-based decision aid for supportive CAM care on pain in childhood cancer. The decision aid was primarily developed as a website for parents, but is considered more than an information website. A decision aid is defined as an evidence-based tool designed to help patients make specific and deliberate choices about available healthcare options [39]. In order to facilitate patient-centered decision-making, the totality of evidence in a decision aid should comprise four pillars: research evidence, practice evidence, patient evidence, and contextual factors [40]. First, the decision aid provides evidence-based information. In addition to high-quality research evidence, it incorporates information from practice (clinical experience), contextual factors (feasibility of implementation), and patient preferences. Second, it is patient-centered and facilitates decision-making by parents of children with cancer, for example helping them to decide whether or not to use CAM as supportive care for their child. 


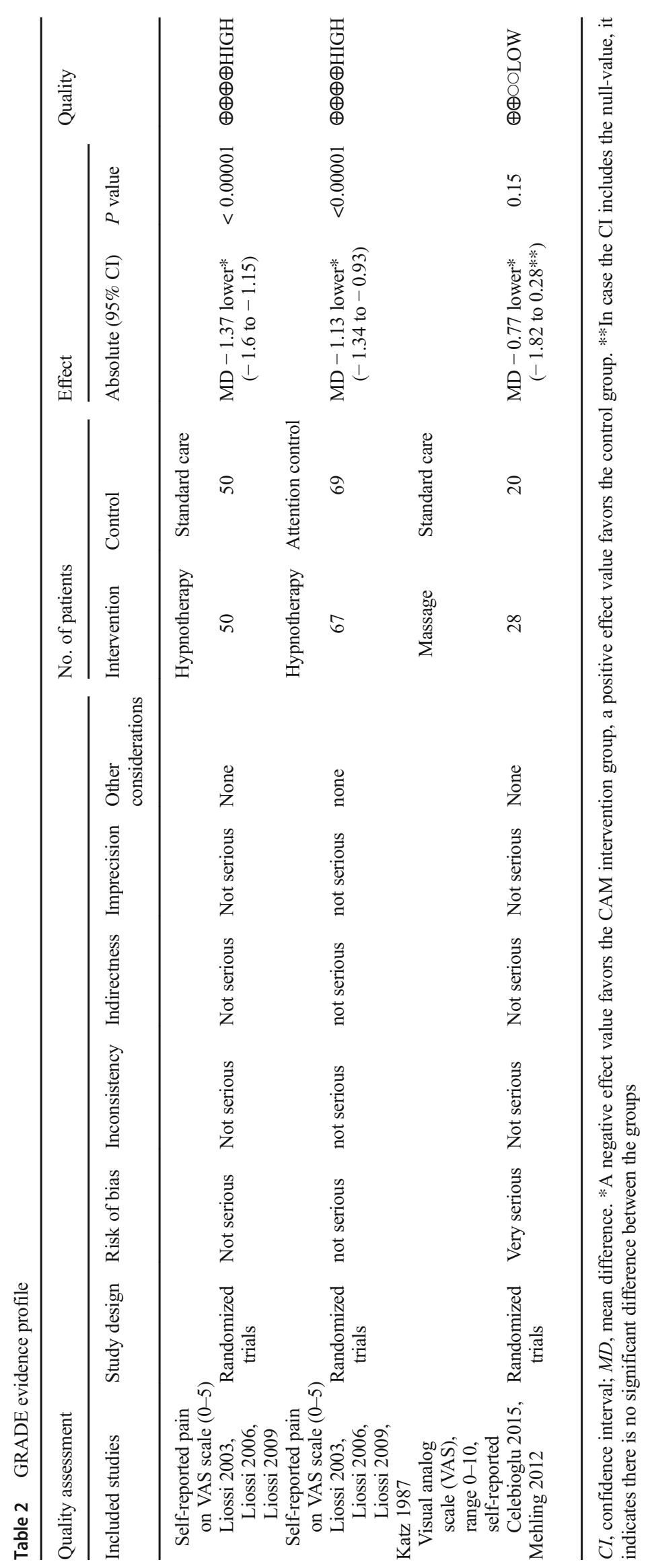


Table 3 Recommendations on CAM use for pain management in children with cancer

\begin{tabular}{lll}
\hline CAM modality & Evidence & Recommendation \\
\hline Hypnotherapy & $\begin{array}{c}\text { There is high-quality evidence } \\
\text { for a positive effect. } \\
\text { No evidence for side effects. }\end{array}$ & $\begin{array}{c}\text { Hypnotherapy is recommended } \\
\text { for the prevention and/or } \\
\text { reduction of procedural pain. }\end{array}$
\end{tabular}

Supplementary information

Mind-body techniques

There is low-quality evidence for an inconsistent effect. No evidence for side effects

Massage

There is low-quality evidence for no effect.

No evidence for side effects

Healing touch

There is low-quality evidence for a positive effect.

Healing touch may be considered for the prevention and/or reduction of pain.

Massage is not recommended for the prevention and/or reduction of pain but may be considered to support general well-being.

ind-body techniques may be considered for the prevention and/or reduction of procedural pain.
Music therapy may be considered for the prevention and/or reduction of procedural pain.
For children of 6 years of age and older; requires concentration and imagination; for procedural and chronic pain, little to no side effects; when the child experiences dizziness, exercises can be performed when laying down; can be guided by a hypnotherapist, a CD or app with exercises or simple exercises can be taught to the parent as to guide their child.

For children of 3-4 years of age and older; mind-body techniques such as breathing and relaxation techniques, meditation, and guided imagery; for procedural pain and pain related to stress and anxiety; supportive care option in combination with physiotherapy; no reported side effects; can be guided by trained nurse or psychologist, a CD or app with exercises or simple exercises can be taught to the parent as to guide their child.

For all ages; in each treatment phase; often young children prefer to receive massage from their parents; no reported side effects; massage is contraindicated for open wounds and skin lesions and low platelet counts; can be provided by physiotherapist, nurse, or simple exercises can be taught to the parent.

For all ages; in each treatment phase; specifically suitable for children that do not like to be touched; no reported side effects; may not align with parents believes or religion; can be provided by trained nurse, therapist or parent can follow a course.

For all ages; in each treatment phase; no reported side effects; can be provided by music therapist, a CD or app.

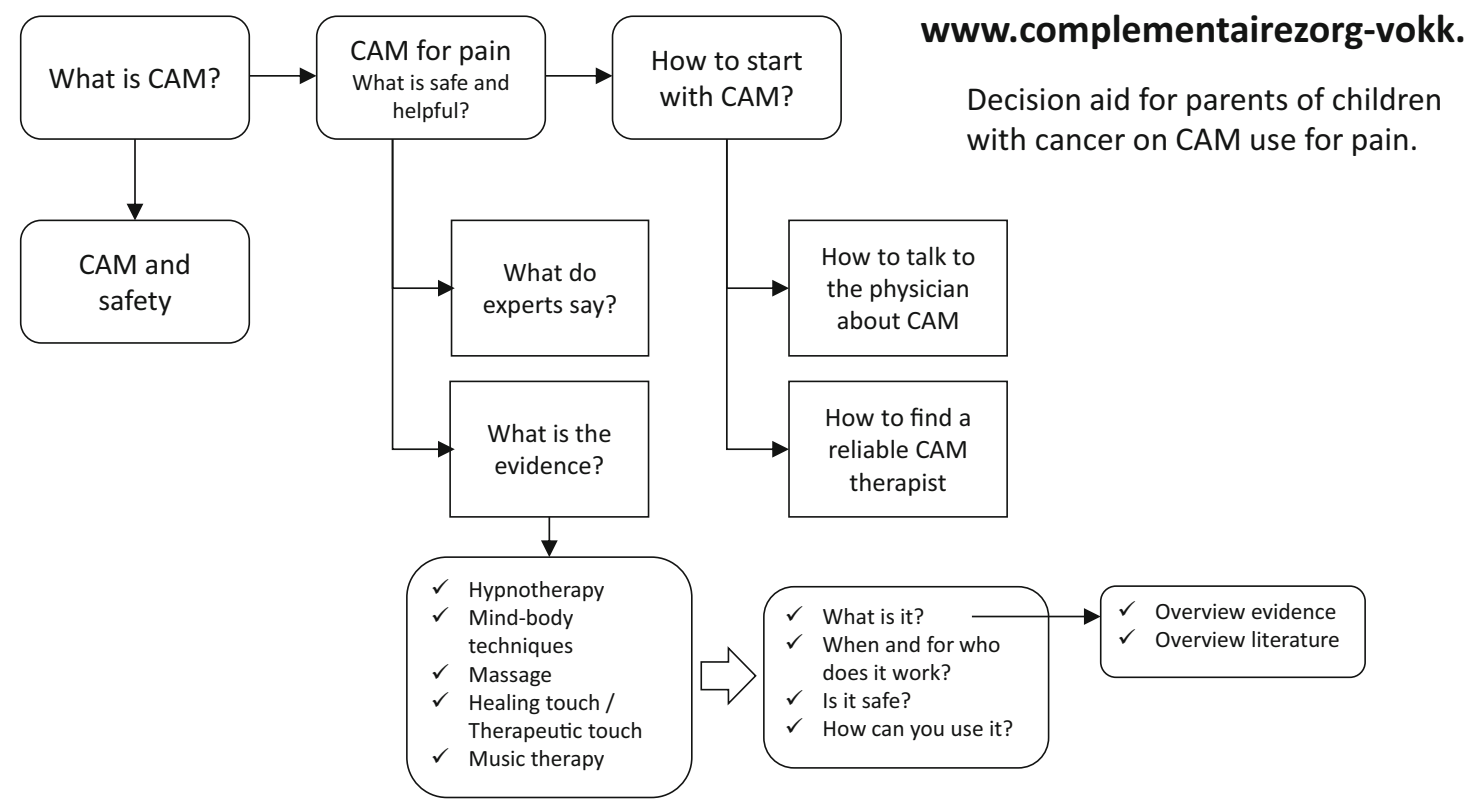

Fig. 3 Visual overview structure decision aid 
Third, it supports parents to recognize possible benefits and harm related to their decision about CAM use for their child. Finally, the decision aid is designed to be helpful for parents in discussions of CAM with healthcare professionals. Although few parents currently discuss CAM use with the treating pediatric oncologist $[7,12,13]$, the majority want to receive information and discuss it with them [7]. The use of the decision aid as a tool has the potential to improve communication on CAM between parents and healthcare providers.

One of the major limitations of the current decision aid is that it is limited to decision-making on CAM modalities for pain relief. The potential effectiveness of the five CAM modalities included in the decision aid, or of other CAM modalities in addressing other complaints than pain, was not investigated. Recently, a RCT in children undergoing hematopoietic stem cell transplants reported that although music therapy did not significantly reduce pain compared with control, it significantly enhanced health-related quality of life [41]. Another major limitation of this study is that it did not evaluate whether the current decision aid actually improved parents' knowledge regarding CAM treatment options, or whether it reduced the decisional conflict stemming from feeling uninformed and unclear about treatment options. Future studies are planned to evaluate the effectiveness of the decision aid for parents in practice and, additionally, to assess its use in communication between parents and healthcare professionals. In the present study, parents wanted information on CAM for a broad spectrum of symptoms related to childhood cancer treatment, i.e., fatigue, anxiety, and pain. This is in line with a previous study, demonstrating that parents perceive high symptom burden in their children with cancer, pain being the most problematic area [42]. Next steps recommended concern expanding the decision aid on CAM for other pediatric cancer-related complaints, as to provide further evidence on how CAM modalities may contribute to reduce the symptom burden in children with cancer. In addition, further investigations are warranted as to disclose potential harmful interactions between CAM remedies and cancer drugs.

A strength of the present study is that the decision aid was developed based on the true needs of parents and was developed by a multidisciplinary team who consider CAM worthy of serious evaluation, so that the perspectives and contextual factors of stakeholders in childhood cancer were taken into account.

In conclusion, an evidence-based decision aid was developed to support parents of children with cancer in making decisions on possible CAM treatment for pain management. Next steps will be to expand the website with evidence for CAM on other childhood cancer-related complaints and to evaluate its use in practice.

Acknowledgments First, we would like to thank Wouter Kollen, Maroeska te Loo, Marc Bierings, Erna Michiels, Hubert Caron, and Arine Vlieger for their scientific and clinical expertise and advice in the different phases of the project. Suzanne van Roijen, Marja van Vliet, and Winnie Schats are acknowledged for their support with the systematic literature review and survey. We are grateful to Erik Loeffen, Lidwien
Daniels, Evelien Otto, Martine van Grotel, Christel Suiker, Juul Coumans, Marie Jose Griffioen, Jacky Gore, and Kees van de Ven for their valuable input and critical feedback. Lastly, we thank Megan Voss, Kara Kelly, Georg J. Seifert, Alfred Längler, Hsing Jou, and Emanuela Portalupi for sharing their clinical experiences with integrative pediatric oncology.

Funding information Open access funding provided by Mid Sweden University. This work was supported by grants from the Foundation Nuts Ohra (grant no. 1404-021) and the Signe and Ane Gyllenberg Foundation.

\section{Compliance with ethical standards}

Conflict of interest The authors declare that they have no conflict of interest.

Ethical approval This project did not involve experiments with patients or study subjects according to the Dutch Medical Research Involving Human Subjects Act (WMO) and was therefore exempt from ethical approval.

Open Access This article is distributed under the terms of the Creative Commons Attribution 4.0 International License (http:// creativecommons.org/licenses/by/4.0/), which permits unrestricted use, distribution, and reproduction in any medium, provided you give appropriate credit to the original author(s) and the source, provide a link to the Creative Commons license, and indicate if changes were made.

\section{References}

1. Trafalska E, Grzybowska K (2004) Probiotics-an alternative for antibiotics? Wiadomosci lekarskie (Warsaw, Poland : 1960) 57(9-10):491498

2. Ladas E, Marjerrison S, Arora B, Hesseling P, Ortiz R, Antillon F, Jatia S, Afungchwi G (2017) Traditional and complementary medicine in pediatric oncology and low-middle income countries: recommendations from the International Society of Pediatric Oncology (SIOP), T\&CM collaborative. 2017. https://doi.org/10.1093/jncimonographs/ $\lg 014$

3. Diorio C, Lam CG, Ladas EJ, Njuguna F, Afungchwi GM, Taromina K, Marjerrison S (2016) Global use of traditional and complementary medicine in childhood cancer: a systematic review. J Glob Oncol 3(6): 791-800. https://doi.org/10.1200/JGO.2016.005587

4. Bishop FL, Prescott P, Chan YK, Saville J, von Elm E, Lewith GT (2010) Prevalence of complementary medicine use in pediatric cancer: a systematic review. Pediatrics 125(4):768-776. https://doi.org/ 10.1542/peds.2009-1775

5. Post-White J, Fitzgerald M, Hageness S, Sencer SF (2009) Complementary and alternative medicine use in children with cancer and general and specialty pediatrics. J Pediatr Oncol Nurs 26(1): 7-15. https://doi.org/10.1177/1043454208323914

6. Magi T, Kuehni CE, Torchetti L, Wengenroth L, Luer S, Frei-Erb M (2015) Use of complementary and alternative medicine in children with cancer: a study at a Swiss university hospital. PLoS One 10(12):e0145787. https://doi.org/10.1371/journal.pone.0145787

7. Singendonk M, Kaspers GJ, Naafs-Wilstra M, Meeteren AS, Loeffen J, Vlieger A (2013) High prevalence of complementary and alternative medicine use in the Dutch pediatric oncology population: a multicenter survey. Eur J Pediatr 172(1):31-37. https:// doi.org/10.1007/s00431-012-1821-6

8. National Cancer Institute. Complementary and Alternative Medicine for Patients. (2019). https://www.cancer.gov/aboutcancer/treatment/cam/patient. Accessed 11 June 2019 
9. Hargrave DR, Hargrave UA, Bouffet E (2006) Quality of health information on the Internet in pediatric neuro-oncology. Neuro-Oncology 8(2):175-182. https://doi.org/10.1215/15228517-2005-008

10. Roth M, Lin J, Kim M, Moody K (2009) Pediatric oncologists' views toward the use of complementary and alternative medicine in children with cancer. J Pediatr Hematol Oncol 31(3):177-182. https://doi.org/10.1097/MPH.0b013e3181984f5a

11. Vlieger AM, van Vliet M, Jong MC (2011) Attitudes toward complementary and alternative medicine: a national survey among paediatricians in the Netherlands. Eur J Pediatr 170(5):619-624. https://doi.org/10.1007/s00431-010-1331-3

12. Gomez-Martinez R, Tlacuilo-Parra A, Garibaldi-Covarrubias R (2007) Use of complementary and alternative medicine in children with cancer in Occidental, Mexico. Pediatr Blood Cancer 49(6): 820-823. https://doi.org/10.1002/pbc.21101

13. Turhan AB, Bor O (2016) Use of herbs or vitamin/mineral/nutrient supplements by pediatric oncology patients. Complement Ther Clin Pract 23:69-74. https://doi.org/10.1016/j.ctcp.2016.03.009

14. Kelly KM (2004) Complementary and alternative medical therapies for children with cancer. Eur J Cancer 40(14):2041-2046. https:// doi.org/10.1016/j.ejca.2004.05.012

15. Ladas EJ (2018) Integrative medicine in childhood cancer. J Altern Complement Med 24(9-10):910-915. https://doi.org/10.1089/acm. 2018.0224

16. Broerse J, Breukers W, Elberse J, Branje D (2012) Patientenparticipatie bij de ontwikkeling van een individueel zorgplan voor mensen met COPD. Athena Instituut

17. Jong MC, van de Vijver L, Busch M, Fritsma J, Seldenrijk R (2012) Integration of complementary and alternative medicine in primary care: what do patients want? Patient Educ Couns 89(3):417-422. https://doi.org/10.1016/j.pec.2012.08.013

18. Paulus PB, Yang H-C (2000) Idea generation in groups: a basis for creativity in organizations. Organ Behav Hum Decis Process 82(1): 76-87. https://doi.org/10.1006/obhd.2000.2888

19. Hsieh H-F, Shannon SE (2005) Three approaches to qualitative content analysis. Qual Health Res 15(9):1277-1288. https://doi. org/10.1177/1049732305276687

20. Shamseer L, Moher D, Clarke M, Ghersi D, Liberati A, Petticrew M, Shekelle P, Stewart LA (2015) Preferred reporting items for systematic review and meta-analysis protocols (PRISMA-P) 2015: elaboration and explanation. BMJ 349:g7647. https://doi.org/10.1136/bmj.g7647

21. Stone JA, Yoder KK, Case EA (2009) Delivery of a full-term pregnancy after TCM treatment in a previously infertile patient diagnosed with polycystic ovary syndrome. Altern Ther Health Med 15(1):50-52

22. Schünemann H, Brożek J, Guyatt G, Oxman A (2013) Handbook for grading the quality of evidence and the strength of recommendations using the GRADE approach. Updated October 2013. The GRADE Working Group, 2013. Available from: www. guidelinedevelopment.org/handbook

23. Guyatt GH, Oxman AD, Vist GE, Kunz R, Falck-Ytter Y, AlonsoCoello P, Schunemann HJ (2008) GRADE: an emerging consensus on rating quality of evidence and strength of recommendations. BMJ 336(7650):924-926. https://doi.org/10.1136/bmj.39489.470347.AD

24. Kramlich D (2014) Introduction to complementary, alternative, and traditional therapies. Crit Care Nurse 34(6):50-56; quiz 57. https:// doi.org/10.4037/cen2014807

25. Celebioglu A, Gurol A, Yildirim ZK, Buyukavci M (2015) Effects of massage therapy on pain and anxiety arising from intrathecal therapy or bone marrow aspiration in children with cancer. Int $\mathrm{J}$ Nurs Pract 21(6):797-804. https://doi.org/10.1111/ijn.12298

26. Katz ER, Kellerman J, Ellenberg L (1987) Hypnosis in the reduction of acute pain and distress in children with cancer. J Pediatr Psychol 12(3):379-394. https://doi.org/10.1093/jpepsy/12.3.379
27. Liossi C, Hatira P (2003) Clinical hypnosis in the alleviation of procedure-related pain in pediatric oncology patients. Int $\mathrm{J}$ Clin Exp Hypn 51(1):4-28. https://doi.org/10.1076/iceh.51.1.4.14064

28. Liossi C, White P, Hatira P (2006) Randomized clinical trial of local anesthetic versus a combination of local anesthetic with self-hypnosis in the management of pediatric procedure-related pain. Health Psychol 25(3):307-315. https://doi.org/10.1037/0278-6133.25.3.307

29. Liossi C, White P, Hatira P (2009) A randomized clinical trial of a brief hypnosis intervention to control venepuncture-related pain of paediatric cancer patients. Pain 142(3):255-263. https://doi.org/10. 1016/j.pain.2009.01.017

30. Mehling WE, Lown EA, Dvorak CC, Cowan MJ, Horn BN, Dunn EA, Acree M, Abrams DI, Hecht FM (2012) Hematopoietic cell transplant and use of massage for improved symptom management: results from a pilot randomized control trial. Evid Based Complement Alternat Med 2012:450150-450159. https://doi.org/ $10.1155 / 2012 / 450150$

31. Nguyen TN, Nilsson S, Hellström A-L, Bengtson A (2010) Music therapy to reduce pain and anxiety in children with cancer undergoing lumbar puncture: a randomized clinical trial. J Pediatr Oncol Nurs 27(3):146-155. https://doi.org/10.1177/1043454209355983

32. Phipps S, Dunavant M, Gray E, Rai S (2005) Massage therapy in children undergoing hematopoietic stem cell transplantation: results of a pilot trial. J Cancer Integr Med 3:62-70

33. Pourmovahed Z, Dehghani K, Sherafat A (2013) Effectiveness of regular breathing technique (hey-hu) on reduction of intrathecal injection pain in leukemic children: a randomized clinical trial. Iran J Pediatr 23:564-568

34. Smith JT, Barabasz A, Barabasz M (1996) Comparison of hypnosis and distraction in severely ill children undergoing painful medical procedures. J Couns Psychol 43(2):187-195. https://doi.org/10. 1037/0022-0167.43.2.187

35. Wong J, Ghiasuddin A, Kimata C, Patelesio B, Siu A (2013) The impact of healing touch on pediatric oncology patients. Integr Cancer Ther 12(1):25-30. https://doi.org/10.1177/1534735412446864

36. Joseph PD, Craig JC, Caldwell PHY (2015) Clinical trials in children. Br J Clin Pharmacol 79(3):357-369. https://doi.org/10.1111/bcp.12305

37. Bond MC, Pritchard S (2006) Understanding clinical trials in childhood cancer. Paediatr Child Health 11(3):148-150

38. Narcis (2018) Princess Máxima Center for pediatric oncology Data Archiving and Networked Services (DANS), KNAW and NWO. https:/www.narcis.nl/organisation/RecordID/ORG1244187/ Language/en. Accessed 26 March 2019

39. Stacey D, Légaré F, Lewis K, Barry MJ, Bennett CL, Eden KB, Holmes-Rovner M, Llewellyn-Thomas H, Lyddiatt A, Thomson R, Trevena L, Cochrane Consumers and Communication Group (2017) Decision aids for people facing health treatment or screening decisions. Cochrane Database Syst Rev 4. https://doi.org/10.1002/ 14651858.CD001431.pub5

40. Clifford AM, Ryan J, Walsh C, McCurtin A (2017) What information is used in treatment decision aids? A systematic review of the types of evidence populating health decision aids. BMC Med Inform Decis Mak 17(1):22-22. https://doi.org/10.1186/s12911-017-0415-7

41. Uggla L, Bonde LO, Hammar U, Wrangsjo B, Gustafsson B (2018) Music therapy supported the health-related quality of life for children undergoing haematopoietic stem cell transplants. Acta Paediatr 107(11):1986-1994. https://doi.org/10.1111/apa.14515

42. Pöder U, Ljungman G, von Essen L (2010) Parents' perceptions of their children's cancer-related symptoms during treatment: a prospective, longitudinal study. J Pain Symptom Manag 40(5):661670. https://doi.org/10.1016/j.jpainsymman.2010.02.012

Publisher's note Springer Nature remains neutral with regard to jurisdictional claims in published maps and institutional affiliations. 